\title{
Simple Infection Control to Radiology in a Dental School in the Northern of Brazil against to COVID-19 Pandemic
}

\author{
Carvalho PL ${ }^{1 *}$, Freitas LVB', Valente LG ${ }^{1}$, Lobato \\ SAS $^{1}$ and Medeiros $\mathrm{J} \mathrm{MF}^{2}$ \\ ${ }^{1}$ Federal University of Para, Brazil \\ ${ }^{2}$ University Brazil, Brazil \\ *Corresponding author: Carvalho PL, School of \\ Dentistry. Federal University of Para. Rua Augusto \\ Correa, 01-Guama, CEP 66075-110, Belem-Para-Brazil
}

Received: February 18, 2021; Accepted: March 12, 2021; Published: March 19, 2021

\begin{abstract}
The purpose of this mini-review is to address a simple protocol, which can be used during radiographic exams at a dental school in Northern Brazil, with few technological resources, to avoid contamination by COVID-19. Protocol proposed for Dental Radiology recommends the cleaning and adequate protection of equipment and surfaces, personal protective measures, which may be obtained by simple procedures such as cleaning and covering of materials and equipment with plastic barriers. The infection control protocol in this Dental School in Northern Brazil, with few technological resources, suggests simple measures to minimize the potential for diseases transmission, including COVID-19.
\end{abstract}

Keywords: Biosafety; Dentistry; COVID-19

\section{Introduction}

In the last twenty years, Coronavirus has been responsible for two pandemics, Severe Acute Respiratory Syndrome (SARS)-CoV, in 2002 and Middle East Respiratory Syndrome (MERS)-CoV in 2012 [1]. In December 2019, an outbreak of pneumonia caused by a new strain of Coronavirus started in the city of Wuhan, Hubei ProvinceChina, and quickly spread to another twenty-four countries. At the end of 2019, the novel Coronavirus was named SARS-CoV-2. This novel Coronavirus produces the disease classified as COVID-19, being the causative agent of a series of pneumonia cases in the city of Wuhan (China) [2]. The nature and perfect measures for clinical management of human cases of SARS-CoV-2 infection have not yet been reported, and there are still many details to be clarified [3].

Biosafety is a set of techniques that should be employed by health professionals to prevent accidents and cross-contamination in clinical environments. The prevention of cross-contamination is a crucial aspect in dental practice. Professionals working in this area should adopt basic prevention routines during their work, since they are responsible for protecting the team, patients, and the oral health care environment, minimizing the risk of disease transmission [4].

In Dental Radiology, the dental radiographer is under constant risk of contracting diseases in of this function in the face of the daily presence of infectious agents. Contamination with the patient's blood or saliva may occur in the equipment used if the asepsis techniques and biosafety standards are not adequately applied [4]. Some microorganisms can stay alive or with activation potential for more than 48 hours and still survive inside the radiographic processing liquids. Thus, the objective of the present mini review is to address a simple protocol, which can be used during radiographic exams at a dental school in Northern Brazil, with few technological resources, to avoid contamination by COVID-19.

\section{Protocol for Dental Radiography}

Dental professionals must be provided with personal protective equipment: surgical cap, medical mask, disposable latex gloves, eye protector and lab coat. The mask should be used to avoid contamination of the mouth and nose by respiratory droplets when the professional operates within one meter of the patient.

Gloves for non-surgical procedures should be worn when there is a risk of hand contact with blood, body fluids, secretions, excretions, mucous membranes, unhealthy skin and contaminated articles or equipment, in order to reduce the possibility of transmission of microorganisms to workers.

Face shields should be used when there is a risk of the dental professionals being exposed to splashes of blood, body fluids and excretions. The use of glasses should be systematic, in clinical settings that include surgical practice and radiographic examination.

The apron should be waterproof and worn during procedures where there is a risk of blood splashes, body fluids, secretions, and excretions, to avoid contamination of the dental radiographer's skin and clothing. It should have long sleeves with elastic cuffs. Furthermore, it must be made with good quality material, non-allergenic and resistant, providing an effective antimicrobial barrier, besides allowing the execution of activities with comfort and being available in several sizes. The dirty apron must be removed and discarded after the procedure and before leaving the service area. After the removal of the apron, hygiene of the hands should be immediately performed to avoid the transmission of microorganisms to the professional, patients and environment. The tube head, position-indicating device, control panel and exposure button must all be covered or disinfected. The bite block, frontal support and chin-support of the panoramic apparatus should be cleaned and decontaminated with alcohol and covered with a plastic barrier, exchanged between each patient.

The next step should be to clean and decontaminate the lead apron and thyroid protector with alcohol. In addition, the cleaning and decontamination with alcohol of all the supporting surfaces should be done before the beginning of the activities, covering them with plastic film. 
The film holder, after their use in the radiographic procedures, should be submitted to chemical disinfection with immersion in enzymatic detergent. The quick wash should be done in running water to remove the disinfectant solution, followed by drying with paper towels. Sterilization should be done in an autoclave with a suitable container, according to the manufacturer's instructions. Dental film and phosphorus plates must be covered with appropriate wrappers, generally those recommended by the manufacturer.

\section{Commentary}

The virus is transmitted through direct contact with respiratory droplets from an infected person (produced during coughing, sneezing). Individuals can also be infected by touching surfaces contaminated with the virus and the face (e.g. eyes, nose, mouth). It is well known that mouthwash prior to treatment reduce the number of microorganisms on surfaces and the environment [5] Thus, it was noted that it is important to define the role of dental services in the management and control of COVID-19 infection, as well as the disposition of instruments and how they should be handled in clinical environments for oral health professionals in Brazil. Cross-contamination may occur frequently during routine intraoral radiographic examination. The $\mathrm{x}$-ray equipment can become contaminated with the oral flora now it is positioned for each exposure by the professional, who handles the image receptor at each exposure [6].

Intraoral image receptors must be packed with plastic material and have their ends sealed with a sealing machine, reducing, or eliminating their contamination [5]. These wrappings should be removed as soon as the films are removed from the oral cavity, bearing in mind that the contaminated glove should not meet the film. Despite the new guidelines for film and film processing, in our geographic region we still use a darkroom equipped with a stopwatch, thermometer, and other resources to ensure processing. Digital radiography should also receive the same attention regarding cross infection control. Intraoral phosphor imaging should be protected with disposable plastic cover before each exposure and exchanged between each patient. After use, ultraviolet light from a scanner is used to disinfect the phosphor-coated plates [7].

\section{Conclusion}

The infection control protocol in this Dental School in Northern Brazil, with few technological resources, suggests simple measures to minimize the potential for diseases transmission, including COVID-19.

\section{References}

1. Xu H, Zhong L, Deng J, Peng J, Dan H, Zeng X, et al. High expression of ACE2 receptor of 2019-nCoV on the epithelial cells of oral mucosa. Int J Oral Sci. 2020; 12: 8.

2. Shamszadeh S, Parhizkar A, Mardani M, Asgary S. Dental considerations after the outbreak of 2019 novel coronavirus disease: a review of literature. Arch Clin Infect Dis. 2020; 15: e103257.

3. Peng X, Xu X, Li Y, Cheng L, Zhou X, Ren B. Transmission routes of n2019nCoV and controls in dental practice. Int J Oral Sci. 2020; 12: 9.

4. Hamedani S, Farshidfar N. The practice of oral and maxillofacial radiology during COVID-19. Oral Radiology. 2020; 36: 400-403.

5. Yu J, Ding N, Chen H, Liu XJ, He W, Dai W, et al. Infection Control against COVID-19 in Departments of Radiology. Acad Radiol. 2020; 27: 614-617.

6. Paul N, Jackie B. DJR and DBT. Recommendations for Diagnostic Imaging during COVID-19 pandemic. 2020

7. Wenzel A, Kornum F, Knudsen MR, Frandsen Lau E. Antimicrobial efficiency of ethanol and 2-propanol alcohols used on contaminated storage phosphor plates and impact on durability of the plate. Dentomaxillofac Radiol. 2013; 42: 20120353 